\title{
The Role of Atypical Chemokine Receptor CCX- CKR (CCRL1) in Human Diseases
}

\author{
Parvin Salimi ${ }^{1}$, Abolghasem Esmaeili2* \\ ${ }^{1}$ MSc Student, Department of Biology, Faculty of Sciences, University of Isfahan, Isfahan, Iran \\ ${ }^{2}$ Department of Biology, Faculty of Sciences, University of Isfahan, Isfahan, Iran
}

\author{
*Correspondence to \\ Abolghasem Esmaeili, Ph.D; \\ Department of Biology, Faculty of \\ Sciences, University of Isfahan, \\ Isfahan, Iran. \\ Email: aesmaeili@sci.ui.ac.ir
}

Published online 26 June 2016

Please cite this article as follows: Salimi $\mathrm{P}$, Esmaeili A. The role of atypical chemokine receptor CCX- CKR (CCRL1) in human diseases. Int J Basic Sci Med. 2016;1(1):29 34. doi:10.15171/ ijbms.2016.07.

\begin{abstract}
The role of chemokines and their receptors have been identified in many biological activities such as immune response and angiogenesis; however, their regulatory ways are under investigation. In recent years, homologous proteins' typical chemokine receptors have been identified. Despite the structural similarity due to changes in a particular motif, they are not able to create signals through $\mathrm{G}$ proteins within the cell; therefore, these chemokine receptors are called silent or unusual. Through binding and internalization and degradation of the chemokines, these receptors regulate the level of ligand in the environment. In this paper, about fifty articles published in the field of the regulatory role of CCX-CKR receptor in some human diseases such as cancer and inflammatory diseases were reviewed. CCX-CKR is one of the chemokine binding proteins that like other silent chemokine receptors, is not able to induce intracellular signaling pathways. Since chemokine network plays a major role in many diseases such as cancer and autoimmune diseases through the impact of CCX-CKR on the level of chemokine environment, preventing intracellular signal creation by these proteins can be considered as a therapeutic target.

Keywords: Chemokine, CCX-CKR, Inflammatory diseases
\end{abstract}

\section{Introduction}

Chemokines are small proteins that regulate cell migration and play an important role in the immune system and processes such as angiogenesis, hematopoiesis, and cell differentiation and development. Chemokines and their respective receptors based on the presence and position of conserved cysteine residues are classified into four different subgroups CXC, CC, C, and $\mathrm{CX} 3 \mathrm{C}$. Chemokine receptors are a kind of $\mathrm{G}$ protein-coupled receptors (GPCRs) that create downstream signaling pathways through G protein. Atypical chemokine receptors are a subfamily of proteins which bind to chemokines. These receptors do not create usual messenger GPCR pathway, but well affect the activity of these receptors with the internalization, degradation, and transferring or delivering chemokines to the usual receptors. ${ }^{1}$ The members of this family include DARC, CCX-CKR, D6, and CXCR7. ${ }^{2}$ CCX-CKR is an atypical chemokine receptor protein that binds with high affinity to hemostatic chemok- ines such as CCL19, CCL21, and CCL25. ${ }^{3}$ Chemokines play an important role in the host response to infection because they are responsible for collecting leukocyte subsets in the sites of pathogen entry. Many inflammatory diseases such as rheumatoid arthritis, inflammatory bowel disease, and asthma are associated with high expression of chemokines. ${ }^{4-7}$ In addition, chemokines are responsible for the migration of cells in lymphoid organs such as the thymus, lymph nodes, and spleen. In processes such as angiogenesis and atherosclerosis, cardiovascular chemokines also play a role. Although the primary function of chemokines is a collection of leukocytes in the site of inflammation, ${ }^{8,9}$ they have biological activities including regulation of cell differentiation, proliferation, survival, and aging. Expression of chemokine and their related receptors have been observed in a variety of human diseases including cancer. ${ }^{10}$ In cancer biology, chemokines, and their receptors, by collecting leukocytes in the location of the tumor, promote surviv-

Copyright (C) 2016 The Author(s); Published by Zabol University of Medical Sciences. This is an open-access article distributed under the terms of the Creative Commons Attribution License (http://creativecommons.org/licenses/by/4.0), which permits unrestricted use, distribution, and reproduction in any medium, provided the original work is properly cited. 
al, proliferation, and spreading of the tumor cells. ${ }^{11}$

In this paper, more than fifty articles from literature were reviewed to investigate briefly the regulatory role of atypical chemokine receptors CCX-CKR in some human diseases such as cancers and inflammatory diseases.

\section{Chemokines}

Chemokines are a large group of cytokines with $6-14 \mathrm{kDa}$ molecular weight and 70 to 125 amino acids. ${ }^{12}$. Chemokines play their roles by binding to seven transmembrane cell surface receptors, coupled with G proteins. ${ }^{13,14}$ They selectively control adhesion, chemotaxis, and activation of many leukocyte populations; that is why they are known as the main regulators of leukocytes trafficking. ${ }^{15}$ Some chemokines are involved in the early stages of inflammatory processes and others are constantly expressed and have important roles in the cell hemostasis and development. Chemokines are permanently produced in lymphoid and non-lymphoid tissues and organs such as skin; in this case, they guide the newly built leukocytes to achieve their proper place. The thymus produces continuously some chemokines, and B cells produce chemokines, as well. ${ }^{16}$ Indirect effects of chemokines are not limited to the immune system. Chemokine family members have also a regulatory role in angiogenesis and wound healing. Usually, inflammatory chemokines are induced by inflammatory responses. Contact with the pathogen or inflammatory cytokines such as tumor necrosis factor (TNF)- $\alpha$ enhances the chemokine production. Chemokines through the induction of leukocyte adhesion to the vascular endothelium cause their migration to the tissues. After migration, leukocytes move toward high concentrations of chemokines. Leukocytes accumulation at sites of infection is directed by chemokines. This is one of the requirements for an appropriate response to infection. ${ }^{17}$ Despite the similarity in the fourth structure of the chemokines, a large difference in the amino acid sequence between the members of this group can be seen. So far 48 different genes encoding the chemokines have been diagnosed. To this, the polymorphisms including editing, $\mathrm{N}$ - and $\mathrm{C}$-terminal regions processing, and chemokines that are coded by the viruses, should be added. Therefore, the number of chemokines may reach to thousands of different types. ${ }^{18,19}$ Chemokines based on the presence and layout of four conserved cysteines at the $\mathrm{N}$-terminus are divided into four groups including $\mathrm{CXC}, \mathrm{CC}, \mathrm{C}$, and CX3C. ${ }^{20,21}$ The nomenclature for chemokine families is based on the time of their genes recognition. However, in addition to the regular designations, all chemokines have other names based on their functions or the origin of the cells that produce them. ${ }^{22}$

\section{Chemokine Receptors}

The chemokines in the target cells interact with cell surface receptors of the GPCR. The molecular weight of these receptors is $40 \mathrm{kDa}$ and they are made up of 340 370 amino acids with acidic N-terminal. Receptor structure is a seven transmembrane $\alpha$-helix that whose $\mathrm{N}$-ter- minal is outside of the cell and C-terminal is inside the cell membrane. These receptors have three loops inside and outside the cell membrane. In each of the four extracellular domains of these receptors, there is a conserved cysteine that binds to the disulfide. ${ }^{23}$ One of the GPCR binding sites on protein $\mathrm{G}$, a highly conserved area among these different types of receptors is called DRYLAIV (or DRY) that includes the amino acids aspartic acid, arginine, tyrosine, leucine, alanine, isoleucine, and valine. This conserved motif area is located between the second transmembrane helix and the second intracellular loop. When the ligand binds to the receptor, the most major motif, namely DRY, is exposed to the protein G and activates it. Ga protein interacts directly with second and third intracellular loops and G $\beta$ subunit. Normally, after chemokine receptor activation by binding and in response to stimulation by ligands and in long-term exposure, desensitization occurs to a received message. In this case, the receptor is internalized and degraded in lysosomal vesicles or returned to the cell surface. The endocytosed receptor determines the severity and duration of response to a received message. Internalization of the receptor is an independent event of $G$ protein. In this process, $G$ protein-coupled receptor kinases (GRKs) are involved that are able to phosphorylate the receptor at the end of C-terminus (the amino acids serine and threonine). The phosphorylation leads to the interaction with $\beta$-arrestin that has the ability to create independent pathways of $G$ protein. After arrestin protein binding, clathrin-mediated endocytosis of receptor is done. Another internalization pathway occurs less frequently by mediating the caveolae and independence of $\beta$-arrestin and clathrin proteins. This event has been observed in CCR2, CCR4, CCR5, DARC, and CCX-CKR receptors. ${ }^{24-26}$ Chemokine receptors similar to chemokines have inflammatory and hemostatic types. In addition, they are classified based on the chemokine that binds to them. CC (CCRs) acceptors identify CC subgroups; whereas CXC (CXCRs) acceptors identify CXC subgroups. Each chemokine can bound to several different receptors and create intracellular signaling pathways. ${ }^{27}$ However, the binding pattern of chemokines is specific and chemokine receptor family members only bind to the same group. This means that CC chemokines do not interact with CXC receptors and they only bind to CCR receptors. However, binding of chemokines such as CCL 26, to the other types of receptors such as CX3CR1, has been observed. ${ }^{28}$

\section{Silent Chemokine Receptors}

Because chemokines play an important role in regulating the immune system, there must be a mechanism that controls the distribution and degradation of them. Atypical chemokine receptors are a subfamily of chemokine binding proteins that are involved in GPCR signaling pathways. They efficiently affect the function of typical chemokine receptors by the internalization and degradation of chemokines. ${ }^{1}$ That is why they are called scavenger proteins, decoy, intercepting receptors, and silent receptors. ${ }^{29}$ 
The main difference between silent chemokine receptors and GPCR receptors is DRYLAIV motif. Any change in DRY motif can dissociate $G$ protein and therefore it could prevent the intracellular signaling pathways. Because of this, cells would not polarize or migrate. ${ }^{30}$ Atypical chemokine receptors together with normal chemokine receptors usually are expressed in wide areas and are rarely found in the blood leukocytes. These receptors also bind with different chemokines; for instance, D6 protein binds to CC and CXC. Naming different kinds of these chemokine receptors, unlike the $\mathrm{G}$ protein binding chemokine, does not follow a general rule. The members of this family include DARC, CCX-CKR, D6, and CXCR7. ${ }^{2}$

\section{CCX-CKR (CCRL1) Proteins}

CCX-CKR is an atypical chemokine receptor protein, which is also called CCR11, (CC-motif) receptor-like 1 (CCRL1), and atypical chemokine receptor 4 (ACKR4). This protein was identified for the first time in 2000 as a possible chemokine receptor in a region of $3 \mathrm{q} 22.1{ }^{31}$ It is made up of 350 amino acids with a molecular weight of $40 \mathrm{kDa}$, which is highly homologous to a taste receptor named PPR1. It has also amino acid sequence similarity with the other chemokine receptors, particularly CCR7 (36\%) and CCR9 (33\%). In this protein, DRYLAIV conserved motif has been changed to DRYVAVT. ${ }^{27,32,33}$ This receptor, like other atypical chemokine receptors, is not able to flux calcium and downstream signaling pathways by binding with a ligand. This atypical chemokine receptor like DARC binds to the CC and CXC chemokine subfamily receptors. However, unlike the DARC and D6, it binds with high affinity to homeostatic chemokines such as CCL19, CCL21, CCL25. ${ }^{33,34}$ This silent chemokine receptor also binds with low affinity to CXCL13 which is a ligand for CXCR5 receptor. ${ }^{8}$ In an effort to locate the protein expression, conflicting results was reported. At the level of mRNA by RT-PCR analysis of human cDNA, it became clear that some populations of immature dendritic cells, T cells, the spleen, ${ }^{35}$ lymph nodes, non-lymphoid tissues such as the heart, kidneys, ${ }^{36}$ brain, and placenta expressed this protein.

However, in Heinzel et al study on CCX-CKR1-EGFP knock-in mice, search for CCX-CKR1-EGFP expression was done. Due to the lack of EGFP expression in the heart, brain, kidneys, and spleen, these results were in contrast to previous studies that had reported its expression in mRNA level. ${ }^{34}$ Despite all studies conducted on these receptors, the cell origin of them is unclear. By making specific antibodies for these proteins, it is expected that in the future studies, more accurate results about the expression of these receptors be announced. ${ }^{37}$ During the internalization of chemokines by CCX-CKR caveolae, pathways are involved instead of dependent $\beta$-arrestin clathrin pathways which are main pathways for common chemokine receptors. The connection of caveolin with transcytosis has been investigated. This suggests the internalization of chemokines from one side of the cell and appearing them from another cell surface. ${ }^{30}$ However, further studies confirming the CCX-CKR is necessary to support this function. Studies conducted in 2013 showed a new role for this protein. ${ }^{1}$ If the cells simultaneously express the chemokine receptor CXCR3 and CCX-CKR, heterodimer receptors could still be generated that could prevent the chemotaxis generated by the CXCR3. The possible causes of this event can be a reduction in CXCR3 ligands access to specific binding sites on the receptor and negative binding cooperativity. ${ }^{1}$

\section{Regulatory Role of CCX-CKR Receptor in Breast Cancer}

Breast cancer is one of the most dangerous and common cancers in women. Survival rates of patients with aggressive and metastatic cancer are low. There is a complex of chemokines and their respective receptors in the microenvironment of tumor that could be a target for the treatment of cancer. ${ }^{38}$ Chemokine/receptor axes of CXCR4/ CXCL12, CCR9/CCL25, CCR7/CCL19 (CCL21), and CXCR5/CXCL13 are the most important axes affecting metastasis, survival, and proliferation of cancer cells. ${ }^{39-41}$ However, chemokine receptor axis alone is not the effective mechanism in cancer cell metastasis. A high expression level of the CCR7 receptor in breast cancer cells have been reported. ${ }^{42}$ Moreover, CCR7/CCL19 (CCL21) axis was observed in the development of many cancers such as lung, gastrointestinal, and melanoma. CCR9/CCL25 axis in addition to breast cancer also plays a role in breast carcinoma, prostate, melanoma, skin, and uterine cancers (Table 1). ${ }^{43,44}$ Studies have shown that a high level of CCL2 chemokine could be found in the serum due to the invasion and metastasis of breast cancer cells. D6 overexpres-

Table 1. Atypical Chemokine Receptors and Their Expression in Different Tissues ${ }^{a}$

\begin{tabular}{lll}
\hline Atypical Chemokine Receptors & Chemokine Ligands & Expression Locations \\
\hline DARC & CCL2, CCL5, CCL7, CCL11,CCL13, & $\begin{array}{l}\text { Erythrocytes, endothelial cells, Purkinjie cells, kidney } \\
\text { epithelial cells, type Il pneumocytes }\end{array}$ \\
& CCL14, CCL17,CXCL5, CXCL6, \\
D6 & CCL2, CCL3, CCL3L1, CCL4,CCL5, & Lymphatic endothelium, lungs, innate B cells, \\
& CCL7, CCL8, CCL11,CCL13, CCL17, & $\begin{array}{l}\text { trophoblasts } \\
\text { CCL22,CCL23, CCL24 }\end{array}$ \\
CXCR7 & CXCL11, CXCL12 & $\begin{array}{l}\text { Somatic cells during development, adult tumour cells } \\
\text { Lymph nodes, thymus, spleen, skin, heart, small } \\
\text { intestine }\end{array}$ \\
\hline
\end{tabular}

See reference 16 . 


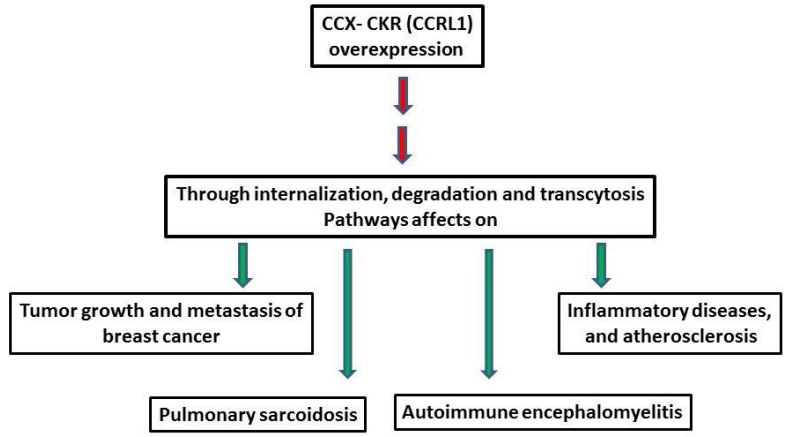

Figure 1. The Role of Atypical Chemokine Receptor CCX-CKR (CCRL1) in Human Diseases.

sion in breast cancer cells by the internalization and degradation of CCL2 prevents the development and invasion of tumor in vitro and inhibits the tumor growth and metastasis of lung and lymph nodes in vivo. ${ }^{45}$ Furthermore, overexpression of D6 or DARC in MDA-MB-231 breast cancer cells internalized CCL2 and destroyed it. ${ }^{46}$ Feng et al created breast cancer cell lines that express CCX-CKR receptor to study the effect of this protein on the cancer. ${ }^{47}$ In contrast to this study, no expression was observed in cancer cells with the ability to metastatize into the lung tissue but its expression was observed in noninvasive cancer. In this research, the relationship between the expression of CCX-CKR and reduction of the growth and metastasis of cancer cells was investigated. ${ }^{47}$ Thus, overexpression of CCX-CKR prevents the spreading and invasion of breast cancer cells in vitro and also inhibits the tumor growth and metastasis of it into the lung and lymph nodes in vivo. In a study, in 98 patients with breast cancer, a significant correlation was observed between the expression of CCXCKR and metastasis of lymph nodes. ${ }^{47}$ Overexpression of CCX-CKR had an indirect correlation with the lymph node metastasis. In addition, it was found that overexpression of this gene is associated with increased length of survival. CCX-CKR role in cancer can be studied from two directions: the effective role in reducing a number of chemokines in cancer development and also the ability to inhibit the chemotaxis or to make different messenger pathways in which as a result of its interaction with other receptors heterodimerization is created. ${ }^{11,48}$

Role of CCX-CKR in Inflammatory Diseases and Atherosclerosis

Atherosclerosis is one of the cardiovascular disorders and the main cause of death. ${ }^{8}$ This disease is associated with the accumulation of leukocytes, particularly macrophages and T cells. MCP chemokines (MCP-1, MCP-4, and MCP-2) play a role in inflammatory diseases, particularly atherosclerosis. In 1999, it was shown that inhibition of macrophage migration into the atherosclerotic lesions, by inhibiting the interaction of MCP-1 with the receptor CCR2, can be helpful in the treatment of atherosclerosis. ${ }^{49}$ In a study, MCP (MCP-1, MCP-4, and MCP-2) receptor was introduced as ligand of CCX-CKR. According to CCX-CKR expression in the heart, it is possible that this protein reduces inflammatory conditions by reducing the environmental chemokine and therefore could be effective in the treatment of heart disease. However, further study did not support this. ${ }^{50}$

The Role of CCX-CKR in Autoimmune Encephalomyelitis The CCX-CKR receptor with the internalization and degradation of CCR7 receptor that is a homeostatic chemokine plays a regulatory role for this receptor. ${ }^{51}$ By producing $\mathrm{CCX}-\mathrm{CKR}^{-1-}$ mice, Comerford et al confirmed the function of these receptors under in vivo conditions. ${ }^{52}$ CCX$\mathrm{CKR}^{-1-}$ rats compared to wild-type ones demonstrated 5 -fold increase in the blood levels of CCL21 protein, and 2 to 3 times increase in CCL19 and CCL21 chemokines in the peripheral lymph nodes. In experimental autoimmune encephalomyelitis model (EAE), in CCX-CKR ${ }^{-1}$ mice compared to wild type, the disease developed faster and more intense, which was associated with an increase in CCL21 in the central nervous system (CNS). In wildtype mice due to the reduction of CCL21 effect, the disease was occurred with delay. Recent reports showed that EAE disease is dependent on the axis of the chemokine receptor CCR7/CCL19-CCL21 that leads to the development of autoimmune diseases in response to Th17.52

The role of CCX-CKR receptor in pulmonary sarcoidosis In another study, an increase in the expression of CCL19 and its receptor CCR7 in pulmonary sarcoidosis was proven. ${ }^{53}$ Pulmonary sarcoidosis is known as a persistent local inflammatory response in alveolar and interstitial space. ${ }^{54}$ Kriegova et al evaluated gene expression level of CCX-CKR in patients with pulmonary sarcoidosis. ${ }^{55}$ In this study, using RT- PCR method, enhancements of mRNA of CCX-CKR gene was shown in patient samples compared to the control. Using local immunocytochemistry, CCX-CKR protein expression in bronchial cells was determined. It was also assessed by flow cytometry fluorescent ligand uptake that showed the ability of ligand internalization by these cells. The CCX-CKR expression by the bronchial cells in the sarcoidosis patients showed the role of this receptor in the modification of immune response and inflammation in the lungs. ${ }^{55,56}$

\section{Conclusion}

In recent years, the identification of atypical chemokine receptors has led to further complexity in the network of chemokine interactions. As mentioned above, these receptors influence the amount of chemokines and their functions through different pathways such as internalization, degradation, and transcytosis. CCL19, CCL21, and CCL25 play an important role in diseases such as autoimmune diseases and cancers by affecting the survival and migration of cells. Interaction of CCX-CKR with these ligands leads to the internalization and degradation of these ligands; therefore their levels would be decreased in the environment. Chemokines expression is controlled at different levels of transcription, translation, and post-translation. Based on the information obtained 
from function and structure of the atypical chemokine receptors, the degradation of this protein on the chemokines would be considered as post-translational control mechanisms. Due to this property, silent chemokine receptors can be used as a model for designing molecules with similar properties, to reduce the level of chemokines as novel therapies. ${ }^{57}$

\section{Ethical Approval}

Not applicable.

\section{Competing Interests}

Authors declare that they have no competing interests.

\section{Acknowledgements}

This article is part of a research project of the University of Isfahan (No. 1041/90).

\section{References}

1. Vinet J, Zwam M, Dijkstra I, et al. Inhibition of CXCR3mediated chemotaxis by the human chemokine receptorlike protein CCX-CKR. Br J Pharmacol. 2013;168(6):13751387. doi:10.1111/bph.12042.

2. Hansell C, Simpson C, Nibbs R. Chemokine sequestration by atypical chemokine receptors. Biochem Soc Trans. 2006;34(Pt 6):1009-1013. doi:10.1042/bst0341009.

3. Salimi P, Esmaeili A, Hashemi M, Behjati M. Endogenous expression of the atypical chemokine receptor CCX-CKR (CCRL1) gene in human embryonic kidney (HEK 293) cells. Mol Cell Biochem. 2016;412(1-2):229-233. doi: 10.1007/s11010-015-2629-2.

4. Lukacs NW, Oliveira SH, Hogaboam CM. Chemokines and asthma: redundancy of function or a coordinated effort? J Clin Invest. 1999;104(8):995-999.

5. Murdoch C, Finn A. Chemokine receptors and their role in inflammation and infectious diseases. Blood. 2000;95(10):3032-3043.

6. Ubogu EE, Cossoy MB, Ransohoff RM. The expression and function of chemokines involved in CNS inflammation. Trends Pharmacol Sci. 2006;27(1):48-55. doi:10.1016/j. tips.2005.11.002.

7. Nibbs RJ, Graham GJ. Immune regulation by atypical chemokine receptors. Nature Rev Immunol. 2013;13(11):815-829. doi:10.1038/nri3544.

8. Charo IF, Ransohoff RM. The many roles of chemokines and chemokine receptors in inflammation. N Engl J Med. 2006;354(6):610-621. doi:10.1038/nri3544.

9. Zabel BA, Rott A, Butcher EC. Leukocyte chemoattractant receptors in human disease pathogenesis. Annual Review of Pathology: Mechanisms of Disease. 2015;10:51-81. doi:10.1146/annurev-pathol-012513-104640.

10. Fernandez EJ, Lolis E. Structure, function, and inhibition of chemokines. Ann Rev Pharmacol Toxicol. 2002;42(1):469499.

11. Chew AL, Tan WY, Khoo BY. Potential combinatorial effects of recombinant atypical chemokine receptors in breast cancer cell invasion: A research perspective (Review). Biomed Rep. 2013;1(2):185-192. doi:10.3892/ br.2013.57.

12. Rollins BJ. Chemokines. Blood. 1997;90(3):909-928.

13. Rossi D, Zlotnik A. The biology of chemokines and their receptors. Ann Rev Immunol. 2000;18(1):217-242. doi:10.1146/annurev.immunol.18.1.217.

14. Graham G, Locati M, Mantovani A, Rot A, Thelen M. The biochemistry and biology of the atypical chemokine receptors. Immunol Lett. 2012;145(1):30-38. doi:10.1146/ annurev.immunol.18.1.217.

15. Epstein FH, Luster AD. Chemokines-chemotactic cytokines that mediate inflammation. $\mathrm{N}$ Engl J Med. 1998;338(7):436-445.

16. Anderson EJR. The role of the CCX-CKR chemokine receptor in immunity and tolerance. University of Glasgow; 2011.

17. Esche C, Stellato C, Beck LA. Chemokines: key players in innate and adaptive immunity. J Invest Dermatol. 2005;125(4):615-628. doi:10.1111/j.0022202x.2005.23841.x.

18. Proost P, Struyf S, Van Damme J. Natural post-translational modifications of chemokines. Biochem Soc Trans. 2006;34(Pt 6):997. doi:10.1042/bst0340997.

19. Colobran R, Pujol-Borrell R, Armengol M, Juan M. The chemokine network. II. On how polymorphisms and alternative splicing increase the number of molecular species and configure intricate patterns of disease susceptibility. Clin Exp Immunol. 2007;150(1):1-12. doi:10.1111/j.1365-2249.2007.03489.x.

20. Zlotnik A, Yoshie O, Nomiyama H. The chemokine and chemokine receptor superfamilies and their molecular evolution. Genome Biol. 2006;7(12):243.

21. Hurson CE. Expression and function of the atypical chemokine receptor CCX-CKR. University of Glasgow; 2011.

22. Chemokine C. Chemokine/chemokine receptor nomenclature. J Interferon Cytokine Res. 2002;22:10671068. doi:10.1089/107999002760624305.

23. Oldham WM, Hamm HE. Heterotrimeric G protein activation by G-protein-coupled receptors. Nat Rev Mol Cell Biol. 2008;9(1):60-71. doi:10.1038/nrm2299.

24. Comerford I, Milasta S, Morrow V, Milligan G, Nibbs R. The chemokine receptor CCX-CKR mediates effective scavenging of CCL19 in vitro. Eur J Immunol. 2006;36(7):1904-1916. doi:10.1002/eji.200535716.

25. Pruenster M, Mudde L, Bombosi P, et al. The Duffy antigen receptor for chemokines transports chemokines and supports their promigratory activity. Nature Immunol. 2008;10(1):101-108. doi:10.1038/ni.1675.

26. Borroni EM, Mantovani A, Locati M, Bonecchi R. Chemokine receptors intracellular trafficking. Pharmacol Ther. 2010;127(1):1-8. doi:10.1016/j. pharmthera.2010.04.006.

27. Watts AO. Dimerization and signaling of the chemokine receptors CXCR3 and CCX-CKR. 2012.

28. Nakayama T, Watanabe Y, Oiso N, et al. Eotaxin-3/CC chemokine ligand 26 is a functional ligand for CX3CR1. J Immunol. 2010;185(11):6472-6479. doi:10.4049/ jimmunol.0904126.

29. Ulvmar MH, Hub E, Rot A. Atypical chemokine receptors. Exp Cell Res. 2011;317(5):556-568. doi:10.1016/j. yexcr.2011.01.012.

30. Comerford I, Litchfield W, Harata-Lee Y, Nibbs RJ, McColl SR. Regulation of chemotactic networks by 'atypical'receptors. Bioessays. 2007;29(3):237-247. doi:10.1002/bies.20537.

31. Gosling J, Dairaghi DJ, Wang Y, et al. Cutting edge: 
identification of a novel chemokine receptor that binds dendritic cell-and $\mathrm{T}$ cell-active chemokines including ELC, SLC, and TECK. J Immunol. 2000;164(6):2851-2856. doi:10.4049/jimmunol.164.6.2851.

32. Khoja H, Wang G, Ng C-TL, Tucker J, Brown T, Shyamala V. Cloning of CCRL1, an orphan seven transmembrane receptor related to chemokine receptors, expressed abundantly in the heart. Gene. 2000;246(1):229-238. doi:10.1016/s0378-1119(00)00076-7.

33. Townson JR, Nibbs RJ. Characterization of mouse CCXCKR, a receptor for the lymphocyte-attracting chemokines TECK/mCCL25, SLC/mCCL21 and MIP-3 $\beta / m C C L 19:$ comparison to human CCX-CKR. Eur J Immunol. 2002;32(5):1230-1241.

34. Heinzel K, Benz C, Bleul CC. A silent chemokine receptor regulates steady-state leukocyte homing in vivo. Proceedings of the National Academy of Sciences. 2007;104(20):8421-8426.

35. Salimi P, Esmaeili A, Hashemi M, Behjati M. Endogenous expression of the atypical chemokine receptor CCX-CKR (CCRL1) gene in human embryonic kidney (HEK 293) cells. Mol Cell Biochem. 2016:412(1):229-233. doi:10.4049/ jimmunol.164.6.2851.

36. Karimian M, Esmaeili A, Behjati M, Hashemi M. Optimization the Production of the Sticky Ends of Human CCRL1 Gene. Journal of Molecular Genetics. 2011;3(1):57.

37. Takatsuka S, Sekiguchi A, Tokunaga M, Fujimoto A, Chiba J. Generation of a panel of monoclonal antibodies against atypical chemokine receptor CCX-CKR by DNA immunization. J Pharmacol Toxicol Methods. 2011;63(3):250-257. doi:10.1016/j.vascn.2010.12.003.

38. Mukherjee D, Zhao J. The Role of chemokine receptor CXCR4 in breast cancer metastasis. Am J Cancer Res. 2013;3(1):46.

39. Smith MC, Luker KE, Garbow JR, et al. CXCR4 regulates growth of both primary and metastatic breast cancer. Cancer Res. 2004;64(23):8604-8612. doi:10.1158/00085472.can-04-1844.

40. Spano J-P, Andre F, Morat L, et al. Chemokine receptor CXCR4 and early-stage non-small cell lung cancer: pattern of expression and correlation with outcome. Ann Oncol. 2004;15(4):613-617.

41. Luker KE, Luker GD. Functions of CXCL12 and CXCR4 in breast cancer. Cancer Lett. 2006;238(1):30-41.

42. Cabioglu N, Yazici MS, Arun B, et al. CCR7 and CXCR4 as novel biomarkers predicting axillary lymph node metastasis in T1 breast cancer. Clin Cancer Res. 2005;11(16):56865693. doi:10.1158/1078-0432.ccr-05-0014.

43. Müller A, Homey B, Soto $\mathrm{H}$, et al. Involvement of chemokine receptors in breast cancer metastasis. Nature. 2001;410(6824):50-56.

44. Kakinuma T, Hwang ST. Chemokines, chemokine receptors, and cancer metastasis. Journal of leukocyte biology. 2006;79(4):639-651. doi:10.1189/jlb.1105633.

45. Wu FY, Ou ZL, Feng LY, et al. Chemokine decoy receptor d6 plays a negative role in human breast cancer. Mol Cancer Res. 2008;6(8):1276-1288.

46. Wang J, Ou Z, Hou Y, et al. Enhanced expression of Duffy antigen receptor for chemokines by breast cancer cells attenuates growth and metastasis potential. Oncogene. 2006;25(54):7201-7211.

47. Feng LY, Ou ZL, Wu FY, Shen ZZ, Shao ZM. Involvement of a novel chemokine decoy receptor CCX-CKR in breast cancer growth, metastasis and patient survival. Cli Cancer Res. 2009;15(9):2962-2970. doi:10.1158/1078-0432.ccr-082495.

48. Cheng X, Hung M-C. Regulation of breast cancer metastasis by atypical chemokine receptors. Cli Cancer Res. 2009;15(9):2951-2953. doi:10.1158/1078-0432.ccr-090141.

49. C Dawson T, A Kuziel W, A Osahar T, Maeda N. Absence of CC chemokine receptor-2 reduces atherosclerosis in apolipoprotein E-deficient mice. Atherosclerosis. 1999;143(1):205-211.

50. Schweickart VL, Epp A, Raport CJ, Gray PW. CCR11 is a functional receptor for the monocyte chemoattractant protein family of chemokines. J Biol Chem. 2000;275(13):9550-9556. doi:10.1074/jbc.275.13.9550.

51. Behjati M, Torktaz I, Mohammadpour M, Ahmadian G, Easton AJ. Comparative modeling of CCRL1, a key protein in masked immune diseases and virtual screening for finding inhibitor of this protein. Bioinformation. 2012;8(7):336-340.

52. Comerford I, Nibbs RJ, Litchfield W, et al. The atypical chemokine receptor CCX-CKR scavenges homeostatic chemokines in circulation and tissues and suppresses Th17 responses. Blood. 2010;116(20):4130-4140.

53. Gibejova A, Mrazek F, Subrtova D, et al. Expression of macrophage inflammatory protein-3 $\beta /$ CCL19 in pulmonary sarcoidosis. Am J Respir Crit Care Med. 2003;167(12):1695-1703. doi:10.1164/rccm.200205-487oc

54. Keane M, Standiford T, Strieter R. Chemokines are important cytokines in the pathogenesis of interstitial lung disease. Eur Respir J. 1997;10(6):1199-1202.

55. Kriegova E, Tsyrulnyk A, Arakelyan A, et al. Expression of CCX CKR in pulmonary sarcoidosis. Inflamm Res. 2006;55(10):441-445.

56. Cheng Z-h, Shi Y-x, Yuan M, Xiong D, Zheng J-h, Zhang $Z$-y. Chemokines and their receptors in lung cancer progression and metastasis. J Zhejiang Univ Sci B. 2016;17(5):342. doi:10.1007/s11033-016-3995-x

57. Parsi B, Esmaeili A, Hashemi M, Behjati M. Transient expression of recombinant ACKR4 (CCRL1) gene, an atypical chemokine receptor in human embryonic kidney (HEK 293) cells. Mol Biol Rep. 2016:43(7):583-589. doi:10.1007/s11033-016-3995-x. 